\title{
Turbulent Flow and Mixing Characteristics in T-Junction*
}

\author{
Masafumi HIROTA** and Hiroshi NAKAYAMA*** \\ ${ }^{* *}$ Department of Mechanical Engineering, Mie University \\ 1577 Kurimamachiya-cho, Tsu-city, Mie 514-8507, Japan \\ E-mail: hirota@mach.mie-u.ac.jp \\ ${ }^{* *}$ R\&D Division, Chubu Electric Power Co., Inc. \\ Kitasekiyama, Odaka-cho, Midori-ku, Nagoya 459-8522, Japan
}

\begin{abstract}
The flow and temperature/concentration fields in the mixing channel have complex three-dimensional and unsteady natures that are accompanied by flow separations and reattachments, longitudinal vortices, and large-scale velocity fluctuations. In this paper, detailed experimental results are presented on turbulent flow and mixing characteristics in a counter-flow type T-junction. The test fluid is water, and a fluorescence tracer is dissolved into the main-channel flow. The velocity and concentration fields are measured by PIV and PLIF with high temporal resolutions. At first, the three-dimensional structures of the velocity and concentration fields and the distributions of the turbulent mass fluxes that are dominant in the mixing process of two fluids with different concentrations are addressed. Then, the POD analyses are applied to the fluctuating velocity and concentration fields to extract their dominant structures. Based on the results of the POD, the turbulent mass transport process between two flows is examined.
\end{abstract}

Key words: Turbulent Flow, Mixing, T-Junction, Turbulent Mass Flux, POD

\section{Introduction}

The mixing T-junctions in which two fluids with different velocities, temperatures and/or concentrations merge and mix are found in various industrial apparatus such as piping systems in power plants, combustion chambers and chemical reactors. The flow and temperature/concentration fields in those channels have complex three-dimensional and unsteady structures that are accompanied by longitudinal vortices, flow separations and reattachments, and large-scale fluctuations. In general, the mixing T-junctions can be classified into two categories as shown in Fig. $1^{(1)(2)}$. One is the cross-flow type T-junction, in which the branch is connected to the straight main channel at right angles. The other is the counter-flow type T-junction in which the mixing channel is connected with the main and counter channels at right angles to form a tee. Most studies on the turbulent mixing in a T-junction conducted to date have focused on the cross-flow type

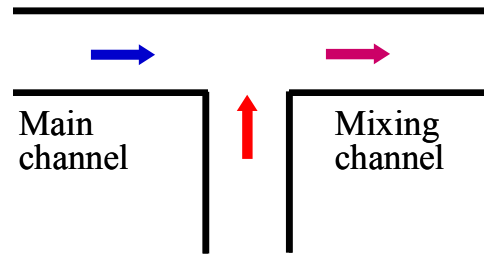

Branch

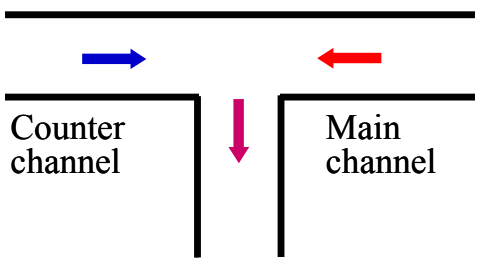

Mixing channel

(a) Cross-flow type T-junction

(b) Counter-flow type T-junction 
T-junction $^{(2)-(7)}$. As for the counter-flow type T-junction, detailed characteristics of the velocity and scalar fields are not clarified yet ${ }^{(8)-(11)}$.

The purpose of this study is to make clear the detailed turbulent flow and mixing characteristics in the counter-flow type T-junction. The test fluid is water, and Rhodamine 6G is dissolved into the fluid in the main channel as a fluorescence tracer. The velocity and concentration distributions in the channel have been measured by PIV and PLIF with high temporal resolutions. In particular, the instantaneous velocity and concentration have been measured simultaneously by combining PIV and PLIF to clarify the characteristics of the turbulent mass fluxes that are dominant in the mass transfer between two fluids ${ }^{(12)}$. The analyses based on the proper orthogonal decomposition ${ }^{(13)(14)}$ have been applied to the fluctuating velocity and concentration fields to extract the dominant flow structures that cause the turbulent mass fluxes in the mixing layer.

\section{Nomenclature}

$\begin{array}{ll}a^{(k)}: & \text { time coefficient of the } k \text { th mode of proper orthogonal decomposition } \\ C: & \text { mean concentration } \\ C_{m}: & C \text { in the main-channel flow before mixing } \\ c: & \text { fluctuating concentration } \\ c^{\prime}: & \text { RMS value of concentration fluctuation } \\ D: & \text { hydraulic diameter of the test channel (=60 mm) } \\ R e: & \text { Reynolds number } \\ U, V, W: & \text { mean velocity components } \\ U_{b}: & \text { bulk velocity in the mixing channel } \\ u, v, w: & \text { fluctuating velocity components } \\ u,, v^{\prime}, w^{\prime}: & \text { RMS values of velocity fluctuations } \\ \overline{u c}, \overline{v c}, \overline{w c}: & \text { turbulent mass fluxes } \\ V R: & \text { velocity ratio of counter to main channel flows } \\ X, Y, Z: & \text { coordinate system (Fig. 1) } \\ \Phi^{(k)}: & \text { eigenfunction of the } k \text { th mode of POD (Eq. (1)) }\end{array}$

\section{Experiments}

A schematic diagram of the experimental apparatus is shown in Fig. 2, and details of the test channel are shown in Fig. 3. The mixing channel is connected to the main and the counter channels at right angles to form a T-junction. All of them have square cross sections of $60 \mathrm{~mm} \times 60 \mathrm{~mm}$ (hydraulic diameter of the channel $D=60 \mathrm{~mm}$ ) and are made of transparent acrylic resin plates to allow an optical access. The test fluid is isothermal water controlled at $25^{\circ} \mathrm{C}$. After flowing through settling chambers and developing channels of $30 D$, the flow in the main channel and that in the counter channel collide head-on in the T-junction. The origin of the coordinate system is at the center of the end wall, thus the entrance of the mixing channel is located at $X / D=1$. Reynolds number of the main-channel flow is kept at 3500 throughout the experiments, and the velocity ratio $(V R)$ of the bulk velocity in the counter channel to that in the main channel is changed from 1.0 to 5.0. In this paper, the results obtained for $V R=3$ (the bulk velocity of the counter flow is three times larger than that of the main flow) are presented because they represent the mixing phenomena observed at $V R>1$.

The velocity field was measured by the time-resolved PIV with a digital high-speed video camera and a continuous $\mathrm{YVO}_{4}$ laser. The fluids in both channels before the $\mathrm{T}$-junction were seeded by Polyamide resin particles whose diameters and density were about $50 \mu \mathrm{m}$ and $1.02 \mathrm{~kg} / \mathrm{m}^{3}$, respectively. The captured images were processed by the direct cross-correlation code with a three-point-Gaussian interpolation for the peak location determination with sub-pixel accuracy ${ }^{(15)}$. We used an own post-processing program to 


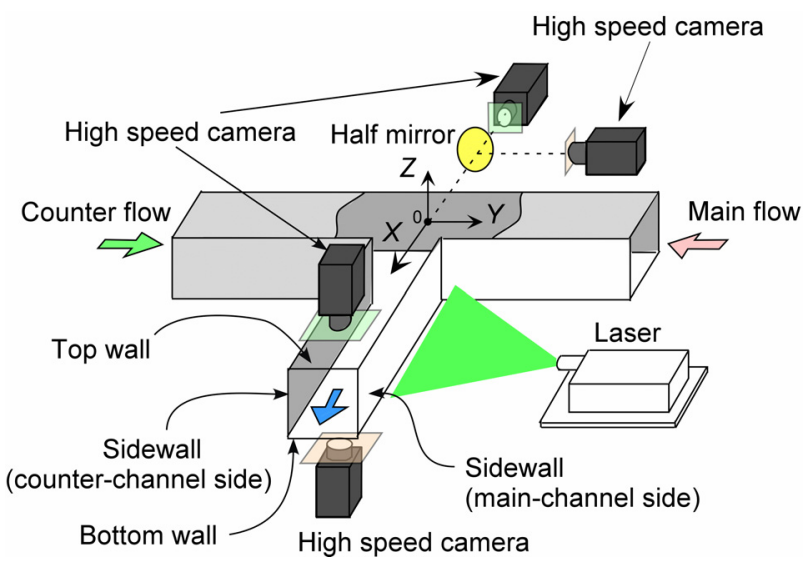

Figure 2 Schematic diagram of test channel and camera arrangement

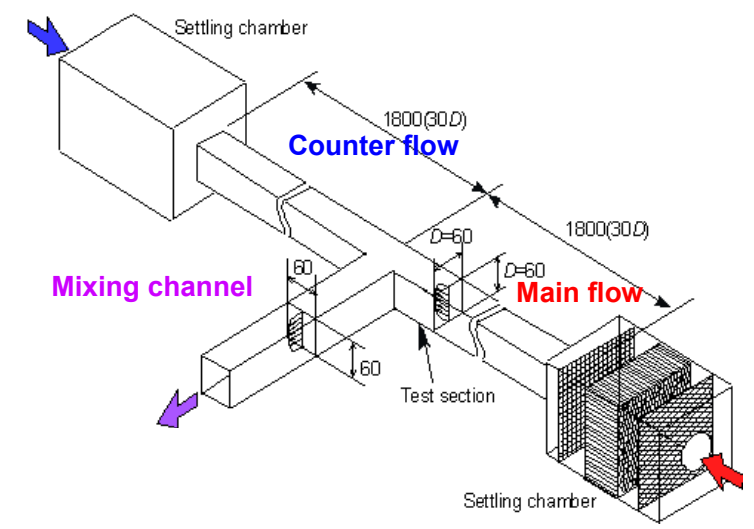

Figure 3 Details of the test channel

calculate the velocity distribution, eliminate the spurious vectors and obtain the statistical flow properties. We also measured the concentration field by the time-resolved PLIF. A dilute solution of Rhodamine $6 \mathrm{G}(0.1 \mathrm{mg} / l)$ was supplied to the main channel flow (low velocity fluid in $V R>1$ ). We made careful calibrations prior/posterior to each experiment run to account for the non-uniform distribution of the light intensity in the laser sheet ${ }^{(12)}$. In PLIF measurement, we used the camera with higher sensitivity to luminous intensity than that used for PIV.

In addition to the individual measurements of the velocity and concentration fields, we measured them simultaneously by combining PIV and PLIF to clarify distributions of the turbulent mass fluxes $\overline{u c}, \overline{v c}, \overline{w c}$, that are dominant to mass transfer between the high and low concentration fluids. In the measurement in the $Y-Z$ plane (cross-sectional plane of the mixing channel), a half mirror was arranged on the extension of the $X$-axis as shown in Fig. 2. An optical filter (sharp-cut filter) was attached in front of each camera lens to separate the scattering light and the fluorescence. For the statistical analysis, the ensemble averages were computed based on about 4000 instantaneous fields. The sampling rate of the high speed camera was 125 frames/second or 250 frames/second. Therefore, the sampling period was 64 seconds or 32 seconds. Since the typical time scale of the large scale velocity fluctuation in the mixing layer was about 7 seconds, the sampling period was long enough to calculate statistics of the turbulence field. These data were normalized by the bulk velocity $U_{b}$ in the mixing channel and by the mean concentration in main channel before the mixing $C_{m}$ that corresponded to the initial concentration difference between two fluids. 


\section{Results and discussion}

\subsection{Characteristics of mean velocity and concentration fields}

In this section, we briefly explain the global characteristics of the mean velocity and concentration fields in the channel ${ }^{(11)}$. Figure 4 shows the mean velocity vectors and primary flow velocity distribution $U / U_{b}$ measured in the symmetric plane of the channel $(Z / D=0)$ for $V R=3$. The flow from the counter channel with a larger momentum (lower side in the figure) is separated at the edge of the T-junction, and a reverse flow region (green region) is formed in the mixing channel along the wall in the counter-channel side.

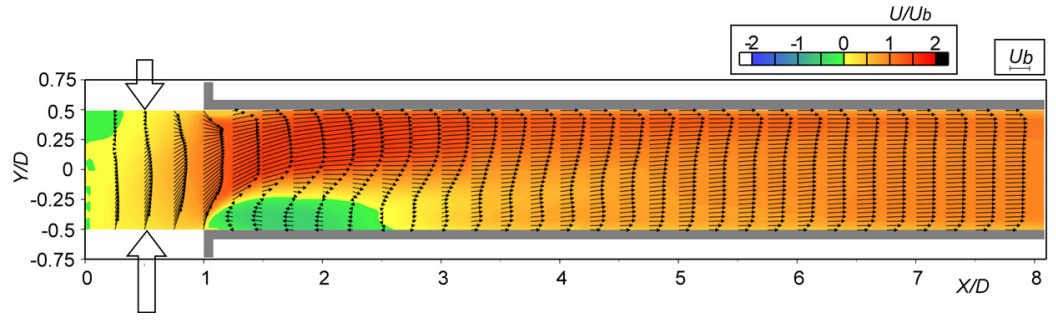

Figure 4 Mean velocity vectors and primary flow velocity distributions in the symmetric plane of the channel $(Z / D=0)$

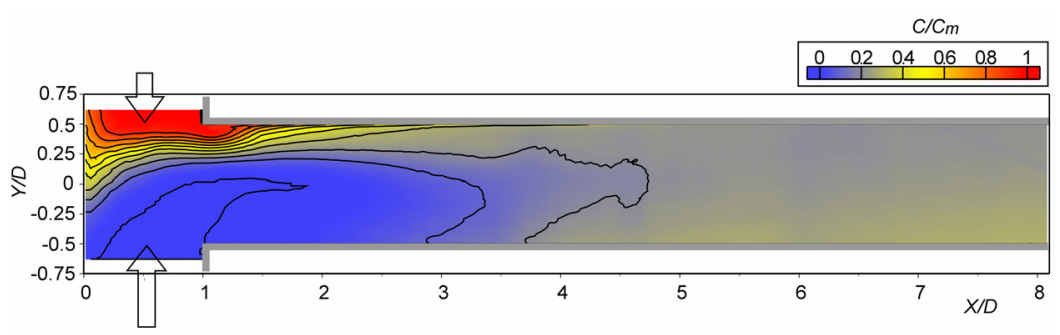

Figure 5 Mean concentration distribution in the symmetric plane of the channel $(Z / D=0)$

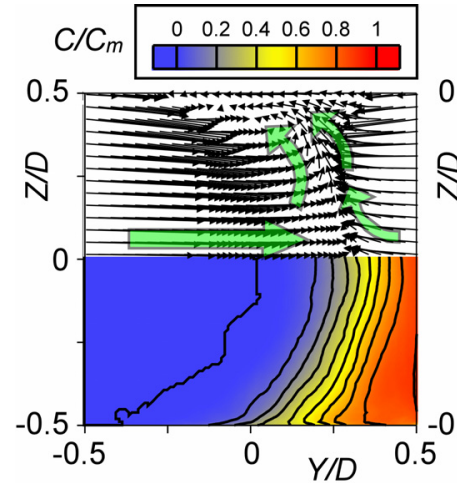

(a) $X / D=1$

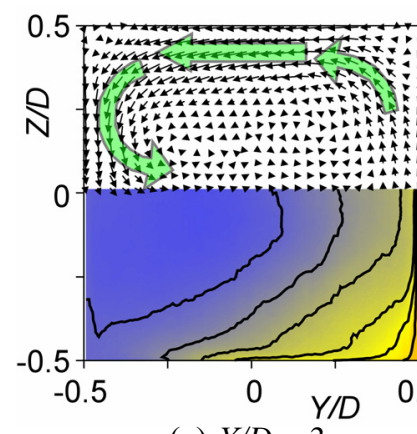

(c) $X / D=3$

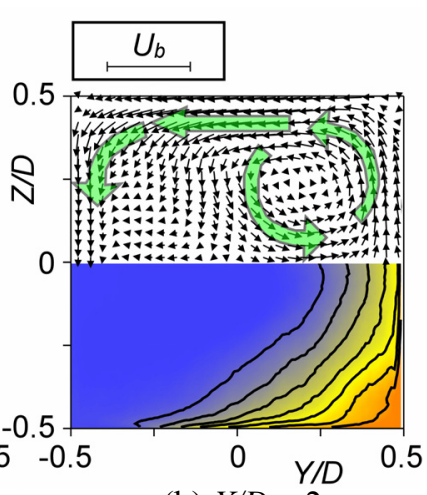

(b) $X / D=2$

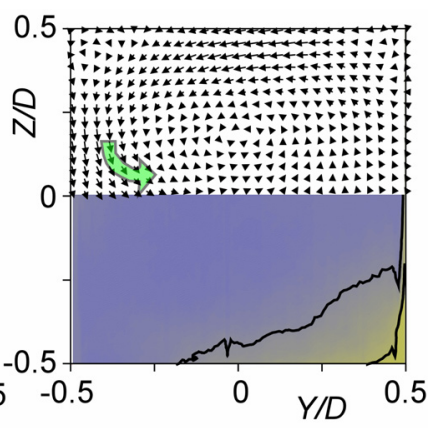

(d) $X / D=5$

Figure 6 Cross-sectional distributions of mean velocity vectors (upper half) and mean concentration (lower half) 
Due to the formation of this separation bubble, the flow in the main-channel side (upper side) is accelerated near the entrance of the mixing channel, but the spanwise non-uniformity of the mean velocity distribution almost disappears near the end of the measuring section.

Figure 5 shows the contour map of the time-averaged local concentration $C / C_{m}$ obtained in $Z / D=0$. In the flow merging region of $0<X / D<1$, the concentration mixing layer (yellow region in the figure) is formed in the main-channel side due to the larger momentum of the counter-channel flow with initially zero concentration. In a further downstream region of $X / D=5 \sim 8$, however, a relatively high concentration region appears in the counter-channel side. This reversal of the high concentration region is caused by the convective transport of mass to this region by a secondary flow as described below.

Figure 6 shows the distributions of the secondary flow vectors (upper figure) and of the time-averaged local concentration (lower figure) obtained at four cross sections in the mixing channel $(X / D=1,2,3$ and 5). At $X / D=1$ that includes the edges of the T-junction, i.e., entrance of the mixing channel, the impingement of the main flow (lower velocity, right-hand side in the map) and the counter flow is observed in the main-channel side. At $X / D=2$, a large longitudinal vortex rotating in the counter-clockwise direction is observed in the main-channel side. It is thought that this vortex is caused by the centrifugal force, which is induced because the flow entering from the counter channel is bent sharply to the $X$-direction in the mixing channel. This vortex pattern and its generation mechanism are similar to those observed in a 90-degree bend ${ }^{(16)}$. Downstream this cross section, the secondary flow proceeding toward the counter-channel side along the top wall appears and longitudinal vortex is not observed clearly. It thus follows that the streamwise scale of the longitudinal vortex mentioned above is quite small, less than $1 D$, in the present channel.

The concentration field is significantly influenced by the secondary flow. In $X / D>2$, the high concentration fluid in the main channel is transported to the counter-channel side by the secondary flow and the concentration mixing layer develops along the bottom wall of the channel. As a result, a high concentration region appears in the counter-channel side after $X / D=5$ as described above. These flow and mixing characteristics of $V R=3$ were qualitative similar to those observed under all the velocity ratios of $V R>2$.

\subsection{Intensities of fluctuating velocities and concentration}

The distributions of turbulence intensities $u^{\prime} / U_{b}$ and $v^{\prime} / U_{b}$ measured in $Z / D=0$ are shown in Figs. 7(a) and 7(b), respectively; the former is the streamwise component, and the latter is the spanwise component. $u$ ' shows very large values in the shear layer around the reverse flow region that is formed along the wall in the counter-channel side. On the other hand, $v$ ' has two peaks; one is observed in the above-mentioned shear layer, and the other appears in the concentration mixing layer observed in Fig. 5. As described in detail later in this paper, the latter is caused by the large-scale wobbling motion of the mixing interface of two flows in the $Y$-direction. After the flow reattachment, $X / D>2.5$, this large $v^{\prime}$ caused by the impingement of two flows is convected to the counter-channel side by the secondary flow, and is merged into $v^{\prime}$ generated in the shear layer around the separation bubble.

Figure 8 shows the intensity of concentration fluctuation $c^{\prime} / C_{m}$ in $Z / D=0 . \quad c^{\prime}$ attains the maximum in the mixing layer, thus it is estimated that this high $c$ ' region is closely related to large $v^{\prime}$ observed in Fig. 7(b). This suggests that the turbulent mass flux $\overline{v c}$ is produced actively in this region, and details are discussed later in this paper.

Next, the cross-sectional distributions of $v^{\prime} / U_{b}$ (horizontal component in the figure) and $w^{\prime} / U_{b}$ (vertical one) measured at $X / D=1,2,3$ and 5 are presented in Figs. 9 and 10, respectively. On the whole the distributions of $v^{\prime}$ are qualitatively similar to those of $w$ '. At $X / D=1$ that corresponds to the entrance of the mixing channel, $v^{\prime}$ and $w^{\prime}$ show large 


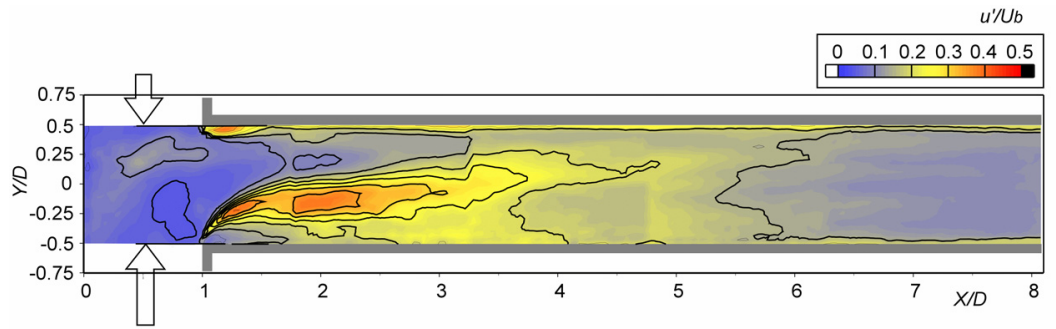

(a) Streamwise turbulence intensity $u^{\prime} / U_{b}$

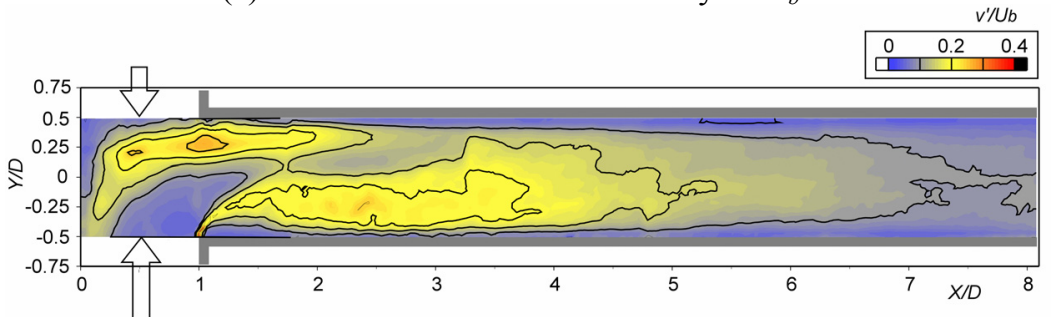

(b) Spanwise turbulence intensity $v^{\prime} / U_{b}$

Figure 7 Distributions of turbulence intensities in $Z / D=0$

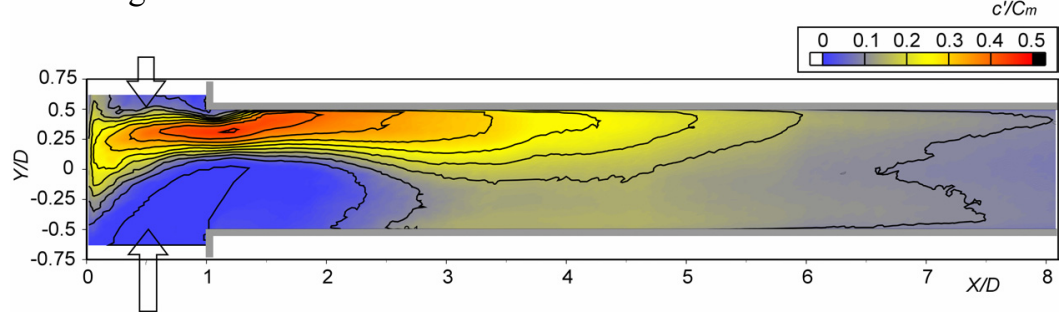

Figure 8 Intensity of concentration fluctuation $c^{\prime} / C_{m}$

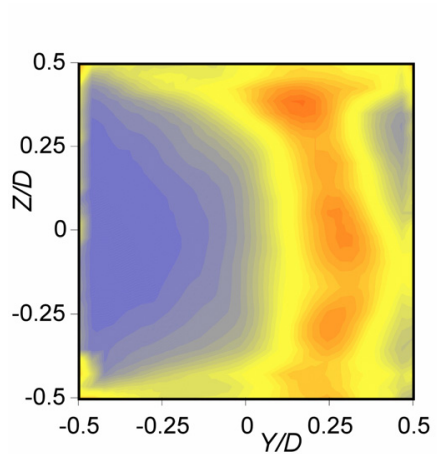

(a) $X / D=1$

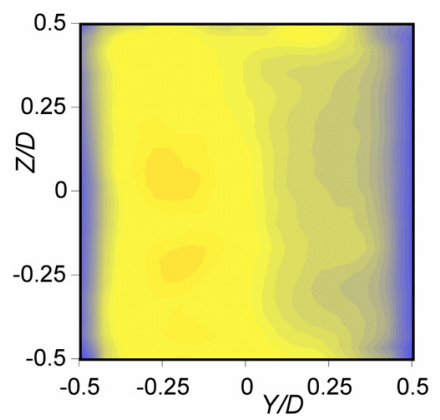

(c) $X / D=3$

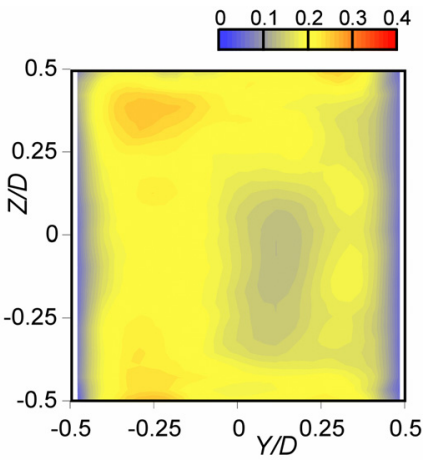

(b) $X / D=2$

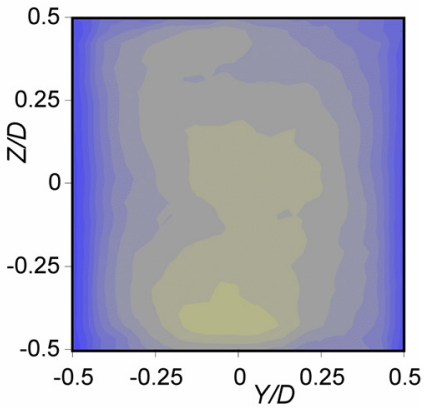

(d) $X / D=5$

Figure 9 Cross-sectional distributions of $v^{\prime} / U_{b}$

values in the mixing layer of two flows and they are almost uniform in the $Z$-direction. From a quantitative viewpoint, however, $w^{\prime}$ is generally smaller than $v^{\prime}$ in this section. This suggests that large $w^{\prime}$ in the mixing layer is caused by the redistribution from $v$. At $X / D=2$ and 3, the peaks in the mixing layer disappear, and relatively large values of $v^{\prime}$ and $w^{\prime}$ are observed in the counter-channel side (left-hand side of the figure). The quantitative 


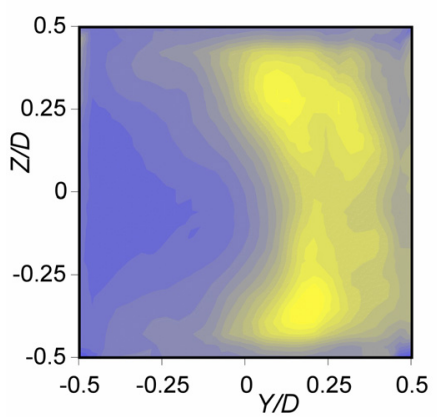

(a) $X / D=1$

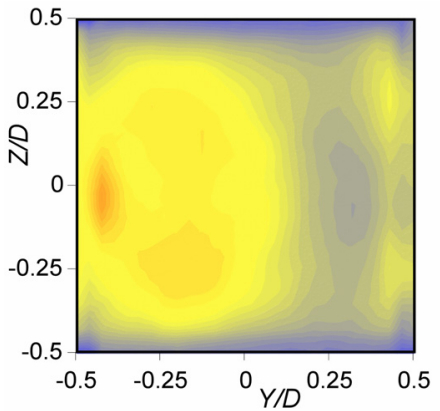

(c) $X / D=3$

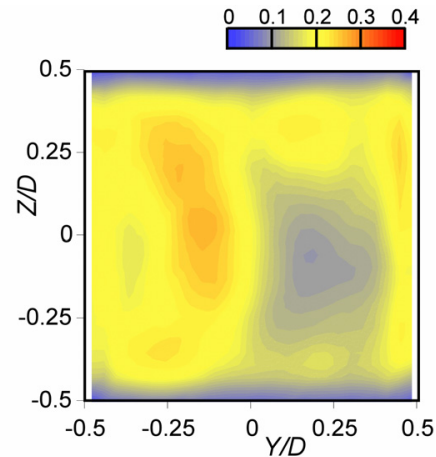

(b) $X / D=2$

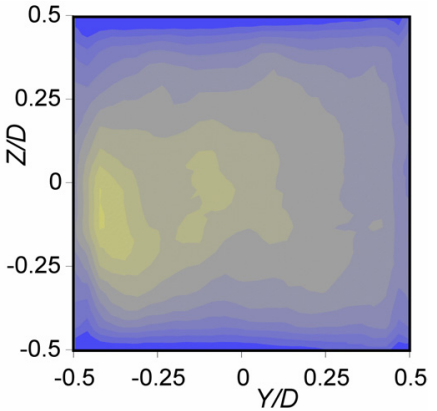

(d) $X / D=5$

Figure 10 Cross-sectional distributions of $w^{\prime} / U_{b}$

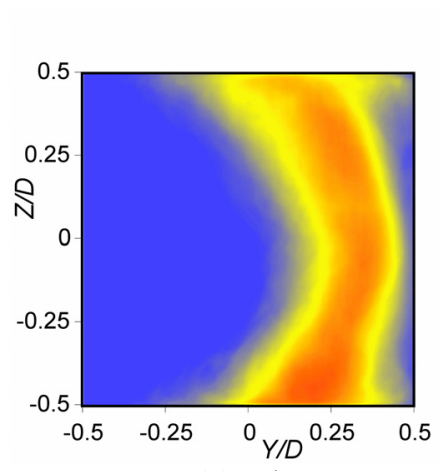

(a) $X / D=1$

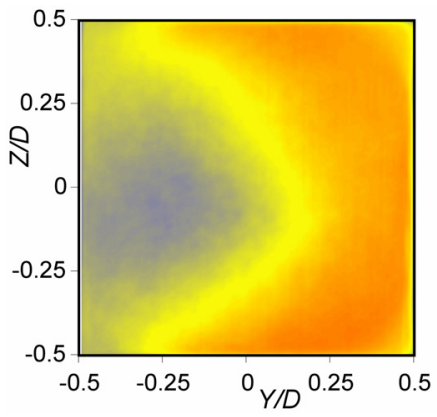

(c) $X / D=3$

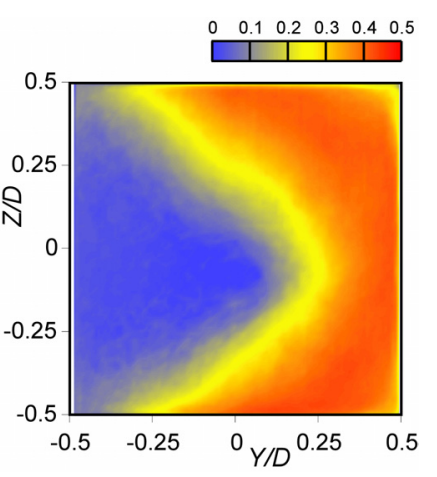

(b) $X / D=2$

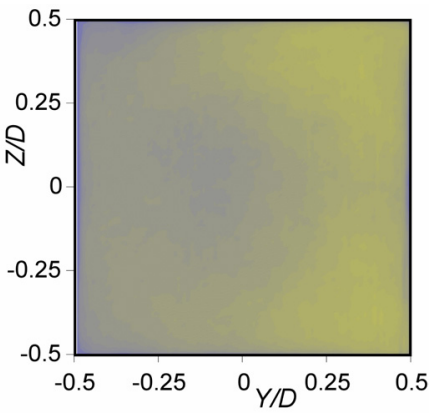

(d) $X / D=5$

Figure 11 Cross-sectional distributions of $c^{\prime} / C_{m}$

difference between $v^{\prime}$ and $w^{\prime}$ as observed in $X / D=1$ is relieved in these cross sections, suggesting these large $v^{\prime}$ and $w^{\prime}$ are caused by the redistributions from $u$ that is produced in the shear layer around the separation bubble.

Figure 11 shows the contour maps of $c^{\prime} / C_{m}$. From a close comparison of this figure with Fig. 6, it is found that the high $c$ ' region develops from the main-channel side to the counter-channel side in phase with the development of the concentration mixing layer. 

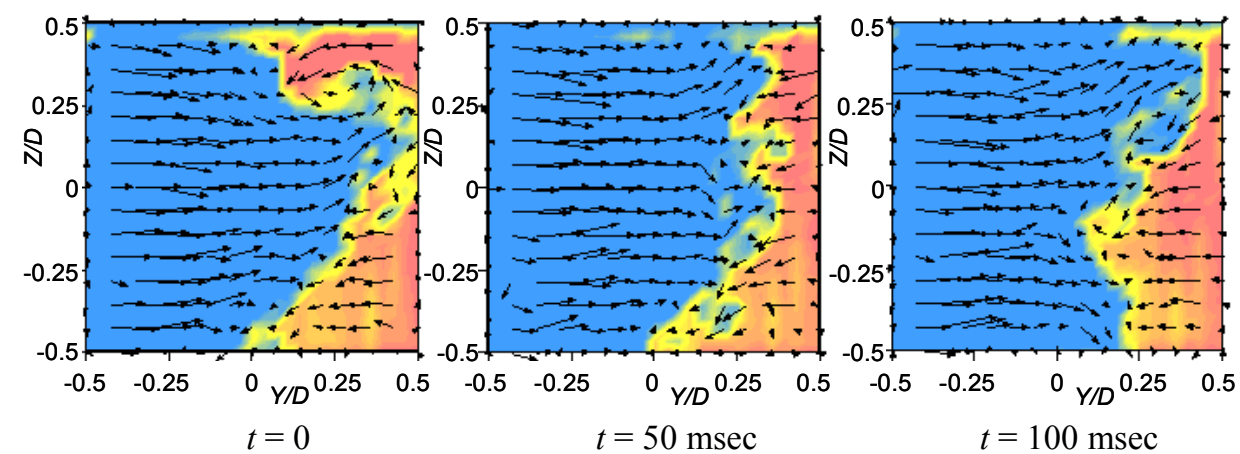

Figure 12 Time evolutions of instantaneous velocity vectors and instantaneous concentration

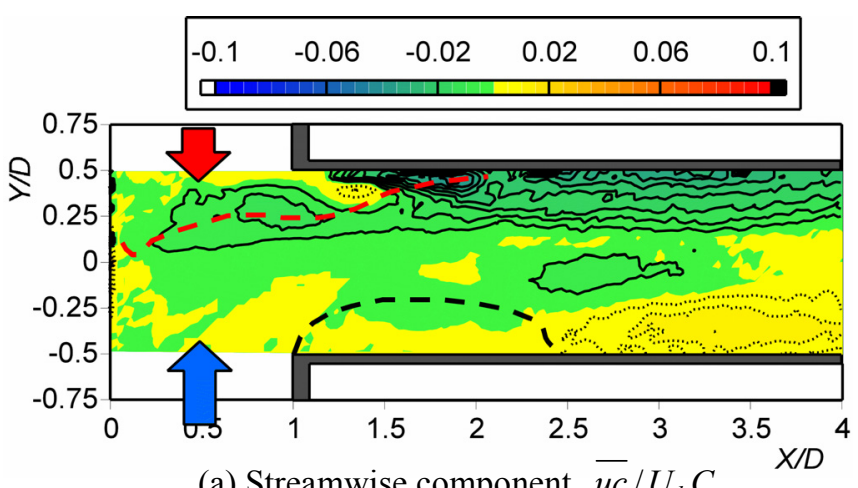

(a) Streamwise component $\overline{u c} / U_{b} C_{m}$

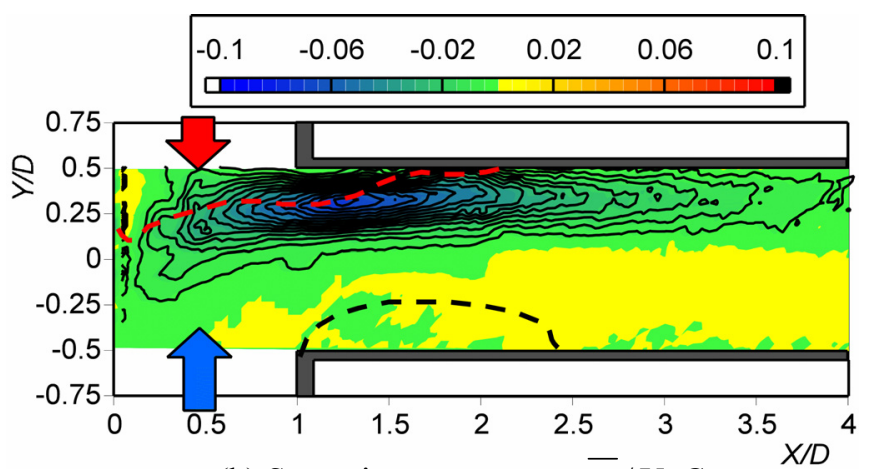

(b) Spanwise component $\overline{v c} / U_{b} C_{m}$

Figure 13 Turbulent mass fluxes measured in the symmetric plane $Z / D=0$

Thus, the distribution of $c^{\prime}$ is qualitatively similar to those of $v^{\prime}$ and $w^{\prime}$ at $X / D=1$, while the difference between the distributions of $c$ ' and turbulence intensities grows in $X / D>2$. This suggests that the turbulent mass transfer in the concentration mixing layer is quite active around $X / D=1$, but it soon deteriorates in further downstream cross sections. In order to examine this point in detail, we made the simultaneous measurements of fluctuating velocities and concentration in $Z / D=0$ and $X / D=1 \sim 3$. Results of the measurements are examined in detail in the next section.

\subsection{Turbulent mass fluxes}

Figure 12 shows examples of the results of the simultaneous measurements of velocity and concentration with combined PIV and PLIF setup. Time-series variations of the instantaneous velocity vectors and concentration measured in the cross section of $X / D=1$ are presented. It is observed that high concentration fluid is conveyed into the low concentration fluid by the instantaneous flow. We then calculated the turbulent mass fluxes based on these data. 


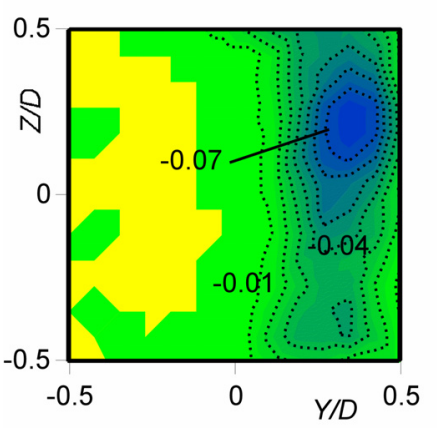

(a) $X / D=1$

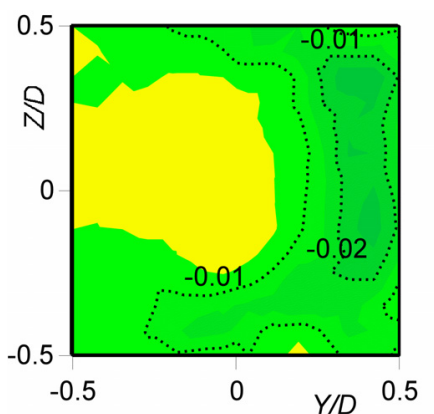

(c) $X / D=2$

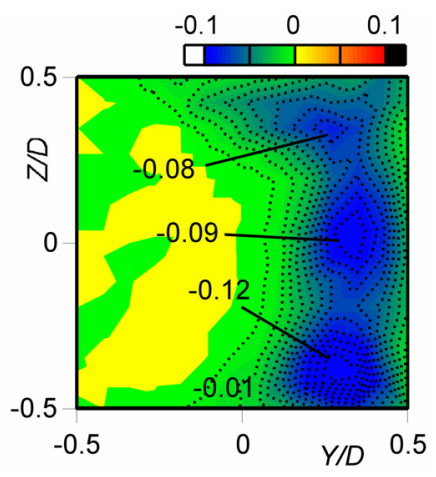

(b) $X / D=1.5$

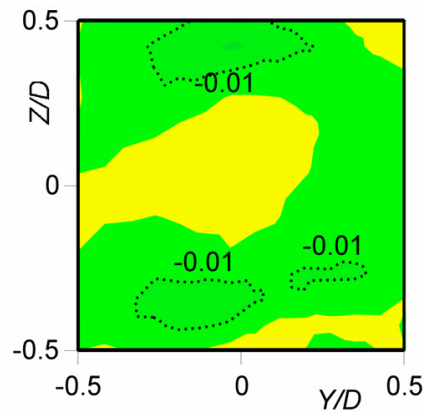

(d) $X / D=3$

Figure 14 Turbulent mass flux in $Y$-direction $\overline{v c} / U_{b} C_{m}$

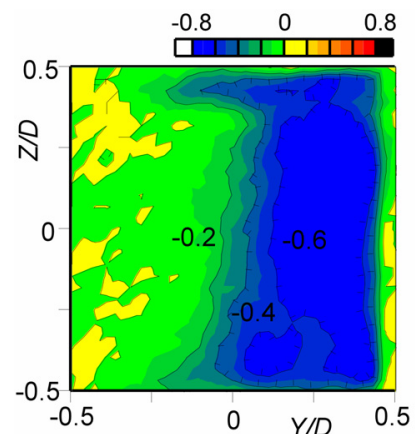

(a) $X / D=1$

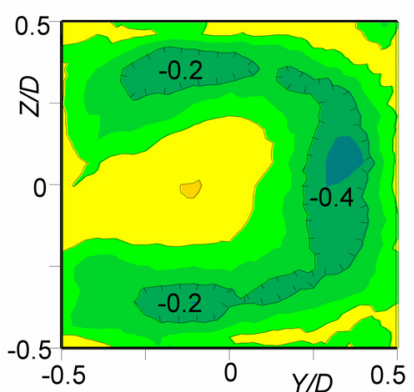

(b) $X / D=3$

Figure 15 Cross-correlation coefficient for $\overline{v c}\left(R_{v c}\right)$

Distributions of the turbulent mass fluxes in the streamwise direction $\overline{u c} / U_{b} C_{m}$ and in the spanwise direction $\overline{v c} / U_{b} C_{m}$ measured in the symmetric plane of $Z / D=0$ are shown in Fig. 13. The red line shows the center of the mixing layer, and black one corresponds to the boundary of the reverse flow region. $\overline{u c}$ shows relatively large negative values in the main-channel side. This negative $\overline{u c}$ can be explained as follows. Near the wall in the main-channel side, $\overline{u v}$ is positive and it follows that positive $v$ causes positive $u$ and negative $c$ assuming the gradient diffusion to the streamwise velocity and concentration. The values of $\overline{u c}$ are quite smaller than those of $\overline{v c}$, thus $\overline{v c}$ is dominant to the turbulent mass transfer in this region. $\overline{v c}$ shows very large values in the mixing layer near the flow merging region and attains peak in $X / D=1 \sim 1.5$. This feature of $\overline{v c}$ distribution is qualitatively similar to that of $c^{\prime}$.

Next, we show the cross-sectional distributions of turbulent mass fluxes in the transverse direction. Figures 14 shows the distributions of $\overline{v c}$ measured in $X / D=1,1.5$, 2 and 3. The distributions of $\overline{v c}$ are qualitatively similar to those of $c$ ' shown in Fig. 11. That is, it shows large values in the main-channel side (right-hand side) at $X / D=1$ and it increases at $X / D=1.5 . \quad \overline{v c}$ is distributed almost uniformly in the $Z$-direction in these 


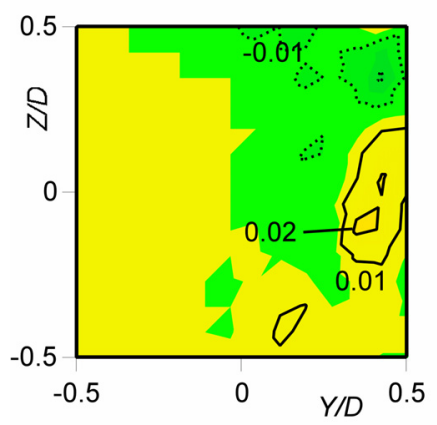

(a) $X / D=1$

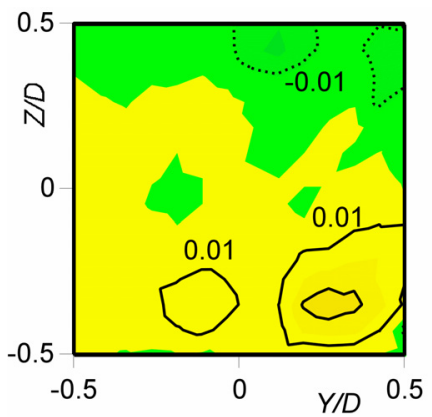

(c) $X / D=2$

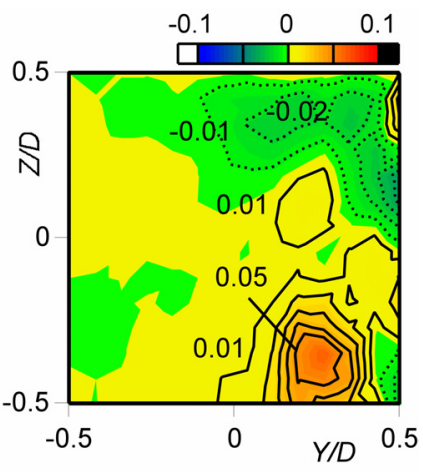

(b) $X / D=1.5$

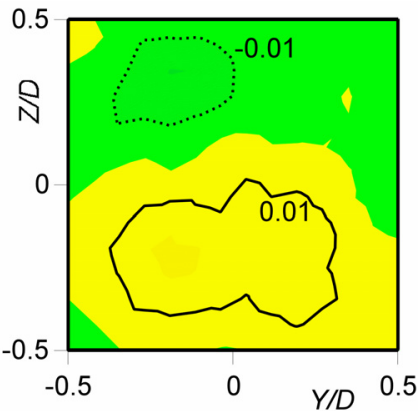

(d) $X / D=3$

Figure 16 Turbulent mass flux in $Y$-direction $\overline{w c} / U_{b} C_{m}$

cross sections. In further downstream locations, the region of relatively large $\overline{v c}$ develops from the main-channel side to the counter-channel side along the top and bottom walls of the channel and $\overline{v c}$ decreases quite quickly; the maximum value of $\overline{v c}$ at $X / D=$ 3 is as small as about $20 \%$ of that at $X / D=1$. The maps of the cross-correlation coefficient $R_{v c}=\overline{v c} / v^{\prime} c$ ' measured in $X / D=1$ and 3 are shown in Fig. 15. The maximum value of $R_{v c}$ in $X / D=3$ is half as much as that in $X / D=1$, thus it follows that $R_{v c}$ decreases in the streamwise direction more slowly than $\overline{v c}$.

Figure 16 shows the distributions of turbulent mass flux in the Z-direction $\overline{w c}$. The distributions of $\overline{w c}$ are dissimilar to those of $\overline{v c}$, and as a whole it is smaller than $\overline{v c}$. $\overline{w c}$ is relatively small at $X / D=1$, then it increases at $X / D=1.5$, and decreases gradually in further downstream cross sections. After $X / D=1.5, \overline{w c}$ shows antisymmetric distributions with respect to the $Y$-axis and its values are comparable to those of $\overline{v c}$. These characteristics of $\overline{w c}$ distributions are closely related to the mean concentration distributions. As shown in Fig. 6, the mixing layer develops from the main-channel side to the counter-channel side along the top and bottom walls of the channel due to the convective mass transport by the secondary flow. Because of this three-dimensional feature of the mean concentration field, the mean concentration gradient $\partial C / \partial Z$ is initially far smaller than $\partial C / \partial Y$ but it once increases after $X / D=1$. At $X / D=3 \partial C / \partial Z$ becomes comparable to $\partial C / \partial Y$, although they are smaller than $\partial \bar{C} / \partial Y$ at $X / D=1$ and 2 . Since the main production of $\overline{w c}$ can be written as a product of $\overline{w^{2}}$ and $\partial C / \partial Z$, the distributions of $\overline{w c}$ reflect the characteristics of $\partial C / \partial Z$ mentioned above.

From the results of the turbulent mass fluxes described so far, it is found that the turbulent mass transfer in the mixing layer is most active around $X / D=1.5$ and $\overline{v c}$ is a dominant component. In the next chapter, we examine the mechanism of the occurrence of the turbulent mass fluxes in this cross section based on the proper orthogonal decomposition (POD) analysis. 


\section{Analysis with proper orthogonal decomposition (POD)}

\subsection{Proper orthogonal decomposition}

We have applied the proper orthogonal decomposition (POD) analyses to the fluctuating velocity components $v$ and $w$, and the fluctuating concentration $c$ measured in $X / D=1.5$ to extract dominant structures that bring about large turbulent mass fluxes there. In this section, we explain the outline of the direct POD used in this study ${ }^{(13)(14)}$.

In PIV and PLIF, instantaneous velocity and concentration can be measured simultaneously at $M$ points in space and $N$ points in time, i.e., a set of $N$ snapshots. We define $f(x, t)$ as a fluctuating velocity or fluctuating concentration in a finite spatial domain $S$, and $f(\boldsymbol{x}, t)$ can be expanded into a finite series of $M$ orthogonal eigenfunctions $\Phi^{(k)}(\boldsymbol{x})$ with time coefficients $a^{(k)}(t)$ as follows.

$$
\begin{aligned}
& f(\boldsymbol{x}, t)=\sum_{k=1}^{M} a^{(k)}(t) \Phi^{(k)}(\boldsymbol{x}) \\
& a^{(k)}(t)=\int f(\boldsymbol{x}, t) \Phi^{(k)}(\boldsymbol{x}) d \boldsymbol{x}
\end{aligned}
$$

The eigenfunction $\Phi^{(k)}(x)$, which represents the spatial structure of the fluctuating velocity or concentration field, is obtained as a solution of the following equation,

$$
\begin{aligned}
& \int_{S} R\left(\boldsymbol{x}, \boldsymbol{x}^{\prime}\right) \Phi^{(k)}\left(\boldsymbol{x}^{\prime}\right) d \boldsymbol{x}^{\prime}=\lambda^{(\boldsymbol{k})} \Phi^{(k)}(\boldsymbol{x}) \\
& R\left(\boldsymbol{x}, \boldsymbol{x}^{\prime}\right)=\frac{1}{N} \sum_{i=1}^{N} f\left(\boldsymbol{x}, t_{i}\right) f\left(\boldsymbol{x}^{\prime}, t_{i}\right)
\end{aligned}
$$

where $R\left(\boldsymbol{x}, \boldsymbol{x}^{\prime}\right)$ is the two-point spatial correlation matrix and $\lambda^{(k)}$ is the eigenvalue of the $k$-th mode that is defined in a decreasing order as $\lambda^{(I)}>\lambda^{(2)}>\lambda^{(3)}>\ldots>0$. The superscript $k$ denotes the eigenmode $(k=1,2,3, \ldots M)$. The total energy of $f(x, t)$, which is denoted as $E$ and defined below, is expressed as a sum of eigenvalues.

$$
E=\int_{S}\left\langle f^{2}(\boldsymbol{x}, t)\right\rangle d \boldsymbol{x}=\sum_{k=1}^{M} \lambda^{(k)}
$$

The contribution ratio made by the $k$-th mode to the total energy, $E_{k}$, is given by the following equation.

$$
E_{k}=\lambda^{(k)} / \sum_{k=1}^{M} \lambda^{(k)}
$$

It follows that the structure expressed by the lower mode has a larger contribution to the total energy. This means that a dominant structure of the fluctuating velocity or concentration field can be reconstructed by several lower modes extracted by the POD. In the following, we show the results of the analyses that are applied to the fluctuating velocity and concentration fields measured simultaneously at $X / D=1.5$, and make clear the mechanism of large turbulent mass flux observed there.

\subsection{First mode of POD}

At first, we discuss the first mode of POD that has been applied to the fluctuating concentration $c$ and the cross-planar fluctuating velocity components $v$ and $w$, all of which were measured simultaneously by combining PLIF and PIV. Figure 17 shows the distributions of the eigenfunction for $c$, denoted as $\Phi_{c}^{(I)}(\boldsymbol{x})$. The contribution ratio of this mode to the total "energy" of fluctuating concentration (Eq. (6)) is about $32 \% . \quad \Phi_{c}^{(l)}(\boldsymbol{x})$ shows relatively large values in the main-channel side (right-hand side), which correspond to large $c^{\prime}$ observed there in Fig. 11. It is remarkable that $\Phi_{c}^{(I)}(\boldsymbol{x})$ is positive near the 
$Y$-axis while it is negative near the channel corners. This distribution of $\Phi_{c}^{(1)}(\boldsymbol{x})$ means that when the instantaneous fluctuating concentration $c$ is positive near the side wall it is negative near the corners at the same instant, and vice versa.

In order to examine the reason for this characteristic distribution of $\Phi_{c}^{(1)}(x)$, we have evaluated the eigenfunctions for $v$ and $w$. The eigenfunctions of the first mode for $v$ and $w$, $\Phi_{v}^{(1)}(\boldsymbol{x})$ and $\Phi_{w}^{(1)}(\boldsymbol{x})$, are shown in Figs. 18(a) and 18(b), respectively. Their contribution ratios are $22 \%$ for $v$ and $18 \%$ for $w$. The distribution of $\Phi_{v}^{(I)}(\boldsymbol{x})$ is qualitatively similar to that of $\Phi_{c}^{(l)}(\boldsymbol{x})$ shown in Fig. 17, except that the sign is just reversed. This result confirms that the fluctuation of the concentration in the main-channel side is closely related to the mass transfer by the fluctuating velocity component $v$, and this produces the large turbulent

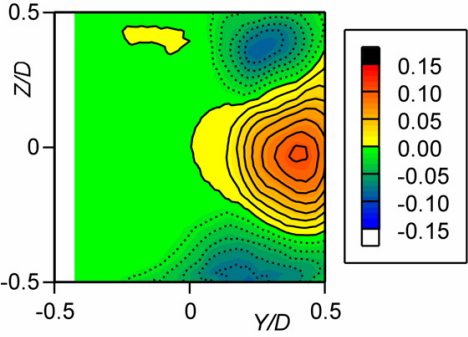

Figure 17 Eigenfunction of 1st mode for $c\left(\Phi_{c}^{(1)}(\boldsymbol{x})\right)$

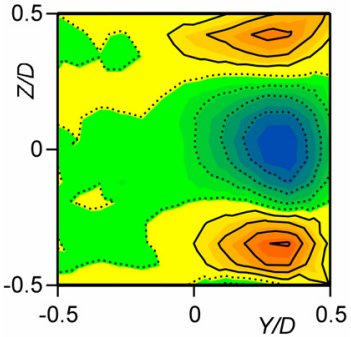

(a) $\Phi_{v}^{(l)}(\boldsymbol{x})$

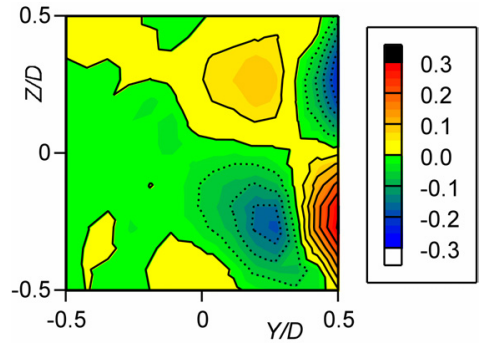

(b) $\Phi_{w}^{(1)}(\boldsymbol{x})$

Figure 18 Eigenfunctions of 1 st mode for $v$ and $w$

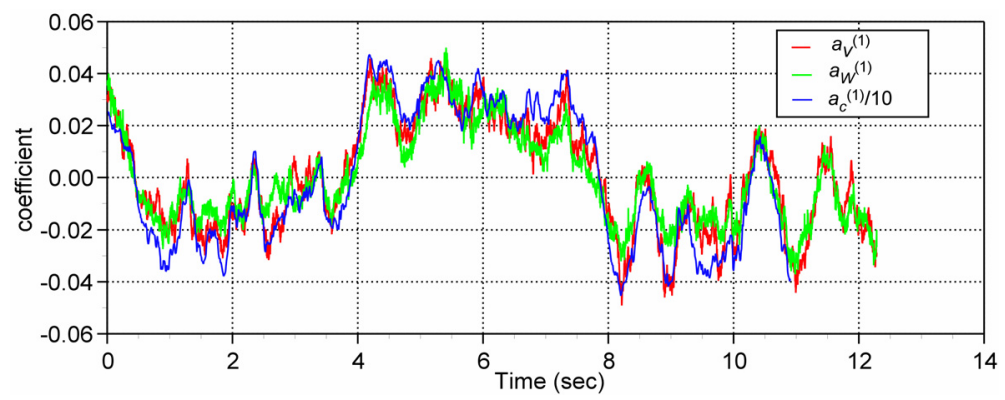

Figure 19 Variations of time coefficients for 1st mode of POD
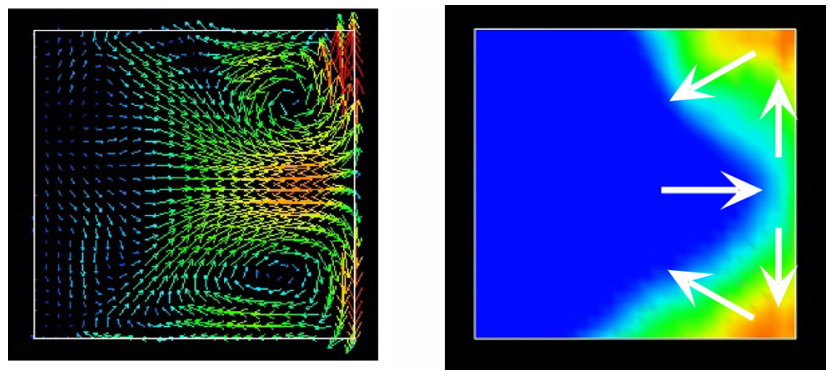

(a) Negative time coefficient
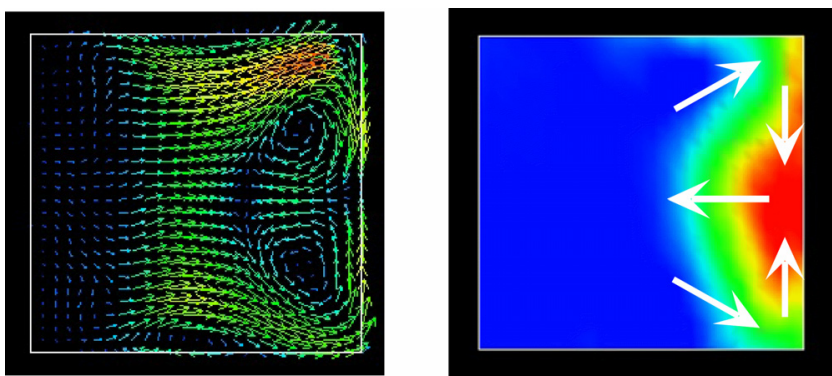

(b) Positive time coefficient

Figure 20 Instantaneous velocity vectors (right) and concentration (left) reconstructed from the 1 st mode 
mass flux $\overline{v c}$ observed in Fig. 14(b). On the other hand, $\Phi_{w}{ }^{(1)}(\boldsymbol{x})$ shows an antisymmetric distribution with respect to the $Y$-axis. It is thought that this characteristic distribution of $\Phi_{w}^{\left({ }^{(l)}\right.}(\boldsymbol{x})$ corresponds to that of $\overline{w c}$, which is negative in $Z / D>0$ and positive in $Z / D<0$ as observed in Fig. 16(b).

The direct (classical) POD we have used in this study is applied to each instantaneous fluctuating components $v, w$ or $c$ separately ${ }^{(17)}$. Therefore, it is not assured that the instantaneous velocity vectors composed from $v$ and $w$, both of which are reconstructed by the first mode, represent a realistic flow structure. As recognized from Eq. (1), however, the simultaneity of $v, w$ and $c$ reconstructed by the first mode is guaranteed if the coefficients $a_{c}{ }^{(l)}(t), a_{v}{ }^{(l)}(t)$ and $a_{w}{ }^{(l)}(t)$ change in a similar manner against time. Thus, we have compared the temporal changes of $a_{c}{ }^{(l)}(t), a_{v}{ }^{(I)}(t)$ and $a_{w}{ }^{(I)}(t)$. Figure 19 shows the results. It is observed that the distributions of these time coefficients agree well with one another. The cross-correlation coefficient of $a_{v}{ }^{(l)}(t)$ and $a_{c}{ }^{(l)}(t)$ was 0.94 and it was smaller than that of $a_{v}{ }^{(l)}(t)$ and $a_{w}{ }^{(l)}(t)$. Thus the simultaneity of $v, w$ and $c$ reconstructed by the first mode of POD is confirmed.

Based on these results, we have reconstructed the instantaneous velocity vectors and concentration distributions from the first mode of POD. Figures 20(a) and 20(b) show the examples of the reconstructed velocity and concentration fields with the time constants of negative and positive values, respectively. In these figures we have added the time-averaged components of velocity and concentration to the reconstructed fluctuating components to approximate the real instantaneous fields. The flow pattern arrows are also superposed on the concentration maps. From these figures, it is understood that the turbulent mass fluxes near the sidewall of the mixing channel is caused by the wobbling motion of the counter-channel flow in the $Y$-direction. When the counter-channel flow with higher velocity and low concentration moves to the main-channel side (right-hand side in the figure), the main-channel flow with high concentration is pushed out to the top and bottom wall sides symmetrically (Fig. 20(a)). Then, the main-channel flow returns to the region near the $Y$-axis when the counter-channel flow moves left (Fig. 20(b)). These flows cause the concentration fluctuation near the wall in the main-channel side, and they produce the turbulent mass fluxes $\overline{v c}$ and $\overline{w c}$ in this region. In particular, $\overline{v c}$ is mainly produced in the region near the $Y$-axis, and $\overline{w c}$ is produced near the corners. Because the first mode of POD has the largest contribution to the total "energy" of the fluctuating fields, it is thought that this flow structure is dominant to the production of the turbulent mass fluxes. In order to confirm this point, we have reconstructed $\overline{v c}$ with the first mode (denoted as $\overline{v^{(1)} c^{(1)}}$ ) and the result is shown in Fig. 21(a). $\overline{v^{(1)} c^{(1)}}$ shows large values in the main-channel side and a region of relatively large $\overline{v^{(1)} c^{(1)}}$ is distributed almost uniformly in the $Z$-direction. These features of $\frac{v^{(1)} c^{(1)}}{w e}$ are similar to those of original $\overline{v c}$. Figure 21(b) shows the distribution of $\overline{w c}$ reconstructed by the first mode $\left(\overline{w^{(1)} c^{(1)}}\right)$, which is qualitatively similar to that of $\overline{w c}$. From these results, it is thought that the first mode is enough to represent the main feature of these turbulent mass fluxes.

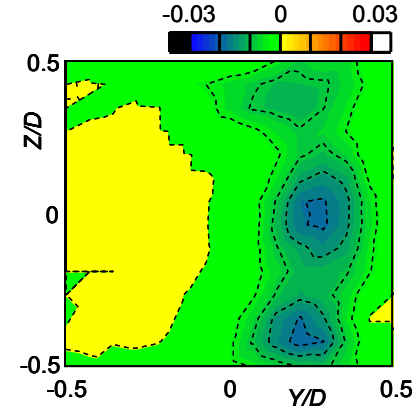

(a) $\overline{v^{(1)} c^{(1)}} / U_{b} C_{m}$

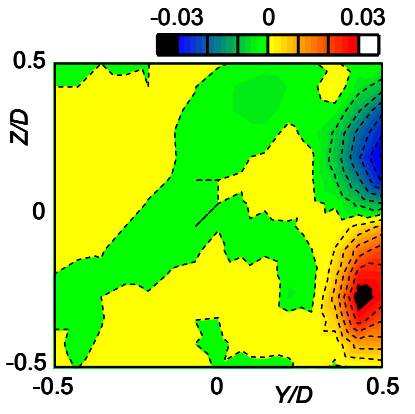

(b) $\overline{w^{(1)} c^{(1)}} / U_{b} C_{m}$

Figure 21 Turbulent mass fluxes reconstructed by the first mode 


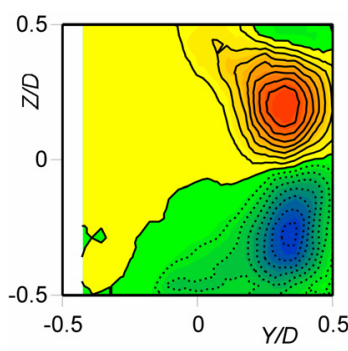

Figure 22 Eigenfunction of 2nd mode for $c\left(\Phi_{c}^{(2)}(\boldsymbol{x})\right)$

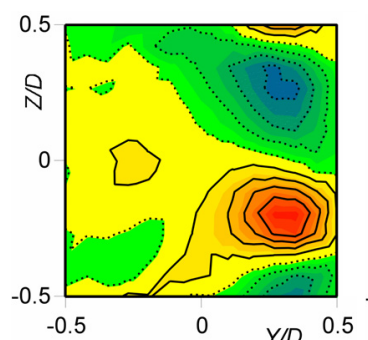

(a) $\Phi_{v}^{(2)}(\boldsymbol{x})$

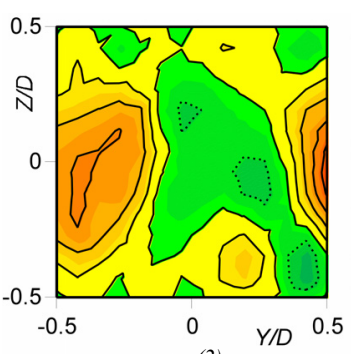

(b) $\Phi_{w}{ }^{(2)}(\boldsymbol{x}$

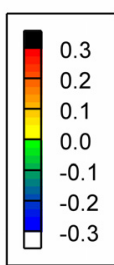

Figure 23 Eigenfunctions of 2 nd mode for $v$ and $w$

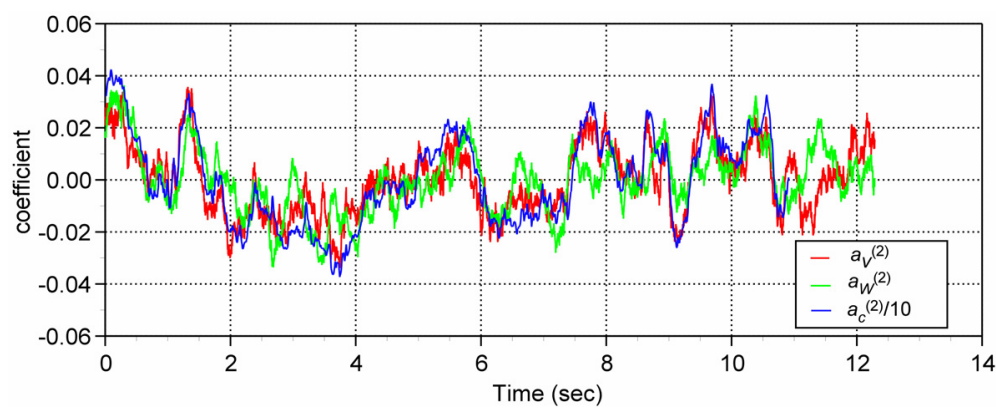

Figure 24 Variations of time coefficients for 2nd mode of POD
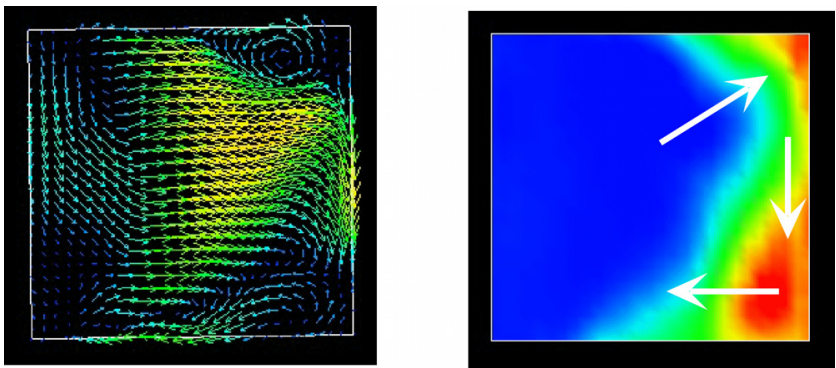

(a) Negative time coefficient
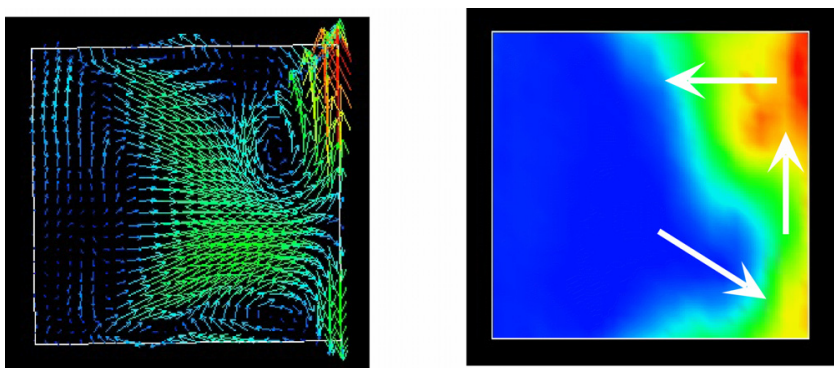

(b) Positive time coefficient

Figure 25 Instantaneous velocity vectors (right) and concentration (left) reconstructed from the 2 nd mode

\subsection{Second mode of POD}

Next, the flow structure expressed by the second mode is addressed. Figure 22 shows the eigenfunction $\Phi_{c}^{(2)}(\boldsymbol{x})$, and Fig. 23 shows $\Phi_{v}^{(2)}(\boldsymbol{x})$ and $\Phi_{w}^{(2)}(\boldsymbol{x})$, the contribution ratios of which are $15 \%, 9 \%$ and $9 \%$, respectively. As is the case with the first mode, $\Phi_{v}^{(2)}(x)$ is qualitatively similar to $\Phi_{c}^{(2)}(\boldsymbol{x})$ and the sign is just reversed. It is remarkable that, in both $\Phi_{v}^{(2)}(\boldsymbol{x})$ and $\Phi_{w}^{(2)}(\boldsymbol{x})$, the regions with relatively large values appear not only in the main-channel side but also in the counter-channel side. As shown in Fig. 7, the velocity fluctuations in the counter-channel side are caused by the flow separation at the edge of the T-junction. These distributions of $\Phi_{v}^{(2)}(\boldsymbol{x})$ and $\Phi_{w}{ }^{(2)}(\boldsymbol{x})$ suggest that the velocity fluctuations in the main-channel side are closely related to this flow separation. 
Figure 24 shows the temporal variations of the time coefficients $a_{c}^{(2)}(t), a_{v}{ }^{(2)}(t)$ and $a_{w}{ }^{(2)}(t)$. Similar to the case of the first mode, the distributions of these coefficients agree well with one another. Therefore, we can reasonably reconstruct the instantaneous velocity vectors and concentration distribution from the eigenfunctions and time coefficients. The reconstructed results are shown in Fig. 25. These results suggest that the concentration fluctuation in the main-channel side is caused by the rotational oscillation of the main-channel flow in the Z-direction. That is, when the counter-channel flow moves toward the upper corner in the main-channel side, the main-channel flow with high concentration is pushed out to the lower corner, and vice versa. This flow structure causes the vertically antisymmetric distributions of $\Phi_{c}^{(2)}(\boldsymbol{x})$, and intensifies $\overline{v c}$ in the corners. It thus follows that the large $\overline{v c}$ observed in the main-channel side in $X / D=1.5$ is caused by the additive effects of the spanwise wobbling motion and the rotationally oscillating motion of fluid.

\section{Conclusions}

We have made simultaneous measurements of instantaneous velocity and concentration in the counter-flow type mixing T-junction to clarify the characteristics of the turbulent mass fluxes. Based on the experimental data, detailed turbulent mass transport process in the mixing layer has been investigated by POD analyses. Main results are summarized as follows.

(1) The flow separates at the edge of the T-junction, and a reverse flow region appears in the mixing channel. Longitudinal vortices are generated in the channel, and the concentration mixing layer shows three-dimensional feature due to the convective transport of mass by those vortices.

(2) The spanwise fluctuating velocity $v^{\prime}$ has two peaks; one appears in the shear layer around the above-mentioned reverse flow region, the other in the mixing layer. The latter is caused by the wobbling motion of the mixing interface of two flows in the spanwise direction. After the flow reattachment, this large $v^{\prime}$ is convected to the counter-channel side by the secondary flow, and is merged into $v^{\prime}$ generated in the shear layer around the separation bubble. The concentration fluctuation $c$ ' attains the maximum in the mixing layer.

(3) The turbulent mass flux $\overline{v c}$ that dominates the turbulent mass transfer in the mixing layer shows large values in the main-channel side in $X / D=1 \sim 1.5 . \quad \overline{v c}$ is distributed almost uniformly in the $Z$-direction in these cross sections. In further downstream locations, the region of relatively large $\overline{v c}$ develops toward the counter-channel side along the top and bottom walls and $\overline{v c}$ decreases quite quickly.

(4) The vertical component $\overline{w c}$ is generally smaller than $\overline{v c}$. It is small at $X / D=1$, then increases at $X / D=1.5$, and decreases gradually in further downstream locations. After $X / D=1.5, \overline{w c}$ shows antisymmetric distributions with respect to the $Y$-axis and its values are comparable to those of $\overline{v c}$.

(5) The POD analyses show that the turbulent mass fluxes near the sidewall of the channel are produced by the superposition of two modes of flow. The first mode is the wobbling motion of the counter-channel flow in the $Y$-direction. The second mode is the rotational oscillation of the main-channel flow in the $Z$-direction.

In this paper, the dominant structure of the flow field is extracted by POD but the dynamic feature of the POD modes is not discussed. The authors estimate that the wobbling motion of the interface of two flows may be related to the flow separation at the edge of the T-junction, and their relevance may be made clear by analyzing the dynamic feature of the POD modes obtained in the symmetric plane of the mixing channel as well as the cross section. This is a subject of the future work. 


\section{References}

(1) Japan Society of Mechanical Engineers ed., Guideline for Evaluation of High-Cycle Thermal Fatigue of a Pipe, JSME S017, 2003 (in Japanese).

(2) Kawamura, T. et al., Experimental Study on Thermal Striping in Mixing Tee with Hot and Cold Water, Proc. of ICONE, 10, ICONE10-22214, pp. 1-4, 2002.

(3) Bruecker, C., Study of the Three-Dimensional Flow in a T-junction Using a Dual-Scanning Method for Three-Dimensional Scanning Particle Image Velocimetry (3-D SPIV), Exp. Thermal Fluid Science, 14, pp. 35-44, 1997.

(4) Wu, H. L. et al., Influence of Sleeve Tube on the Flow and Heat Transfer Behavior at a T-junction, Int. J. Heat and Mass Transfer, 46, pp. 2637-2644, 2003.

(5) Fukushima, N, et al., Numerical and Experimental Study on Turbulent Thermal Mixing in a T-junction Flow, Proc. 6th ASME-JSME Joint Thermal Engineering Conference, TED-AJ03-582, 2003, in CD-ROM.

(6) Hirota, M., et al., Three-Dimensional Structure of Turbulent Flow in Mixing T-junction, JSME International Journal, Ser. B, 49-4, pp. 1070-1077, 2006.

(7) Hirota, M., et al., Experimental study on turbulent mixing process in cross-flow type T-junction, Int. J. Heat and Fluid Flow, 31, pp. 776-784, 2010.

(8) Stull, F.D., et al., Investigation of a Dual Inlet Side Dump Combustor Using Liquid Fuel Injection, J. Propulsion, 1, pp. 83-88, 1985.

(9) Liou, T.-M, et al., Effect of Inlet Guide-Vane Number on Flowfields in a Side-dump Combustor, Experimental Thermal Fluid Science, 24, pp. 11-23, 2001.

(10) Hu. L.-W. and Kazimi, M. S., LES benchmark study of high cycle temperature fluctuations caused by thermal striping in a mixing tee, Int. J. Heat and Fluid Flow, 27, pp. 54-64, 2006.

(11) Hirota, M. et al, Experimental Study on Turbulent Flow and Mixing in Counter-flow Type T-junction, J. Thermal Science and Technology, 3, pp. 147-158, 2008.

(12) Law, A.W-K. et al., Measurement of Mixing Process with Combined Digital Particle Image Velocimetry and Planar Laser Induced Fluorescence, Exp. Thermal Fluid Science, 22, pp. 213-229, 2000.

(13) Graftieaux, L., et al., Combining PIV, POD and Vortex Identification Algorithms for the Study of Unsteady Turbulent Swirling Flows, Meas. Sci. Technol., 12, pp. 1422-1429, 2001.

(14) Patte-Rouland, B. et al., Flow Analysis of an Annular Jet by Particle Image Velocimetry and Proper Orthogonal Decomposition, Meas. Sci. Technol., 12, pp. 1404-1412, 2001.

(15) Raffel, M., et al. Particle Image Velocimetry, Springer-Verlag, Berlin, pp. 129-134, 1998.

(16) Sudo, K. et al., Experimental Investigation on Turbulent Flow in a Square-Sectioned 90-degree Bend, Exp. Fluids, 30, pp. 246-252, 2001.

(17) Hilberg, D., et al., The Application of Classical POD and Snapshot POD in a Turbulent Shear Layer with Periodic Structures, Applied Scientific Research, 53, pp. 283-290, 1994. 